\title{
Chlorhexidine mouthwash reduces plaque and gingivitis
}

\author{
Abstracted from \\ Van Strydonck DA, Slot DE, Van der Velden U, Van der Weijden F. \\ Effect of a chlorhexidine mouthrinse on plaque, gingival inflammation and \\ staining in gingivitis patients: a systematic review. / Clin Periodontol 2012; 39: 1042-1055. \\ doi: 10.1111/j.1600-051X.2012.01883.x. Epub 2012 Sep 7. PubMed PMID: 22957711. \\ Address for correspondence: Daniëlle A Van Strydonck, Department of Periodontology, \\ Academic Centre for Dentistry Amsterdam (ACTA), Amsterdam, The Netherlands. E-mail: d.v.strydonck@telenet.be
}

\section{Question: Is chlorhexidine mouthrinse effective in reducing plaque and gingival inflammation compared with placebo?}

Data sources Medline, Embase and Cochrane Central Register of Controlled Trials (CENTRAL) databases were searched along with the reference lists of all selected studies. Only English language studies were included.

Study selection Randomised controlled clinical trials comparing chlorhexidine (CHX) to placebo/control mouthrinses for oral hygiene in studies of at least four weeks duration were included.

Data extraction and synthesis Screening, selection and data abstractions were conducted independently by two reviewers. Where possible meta-analysis of weighted mean differences was carried out using fixed or random effects models where appropriate.

Results Thirty studies were included, with 13 studies contributing to the meta-analysis. The meta-analysis found significant differences favouring $\mathrm{CHX}$ for a range of indices; the Plaque Index Silness \& Löe, Plaque-Index Quigley \& Hein (PIQH), the Gingival Index (GI), Papillary Bleeding Index, Bleeding on Marginal Probing and the Lobene Stain Index. Relative to control, the reduction with $\mathrm{CHX}$ for plaque was $33 \%$ and for gingivitis $26 \%$. CHX rinsing groups demonstrated significantly more staining.

Conclusions In gingivitis patients, $\mathrm{CHX}$ mouthrinses together with $\mathrm{OH}$ versus placebo, or control mouthrinse provide significant reductions in plaque and gingivitis scores, but a significant increase in staining score.

\section{Commentary}

Prevention of periodontal diseases is based on supragingival biofilm control, with mechanical plaque control as first line option. ${ }^{1}$ However, mechanical control has limitations and chemical control may be relevant. Among the evaluated agents for chemical control, chlorhexidine ( $\mathrm{CHX}$ ) is the gold standard, as supported by a large body of evidence, produced since $1970 .^{2}$ In the commented review, the proposed justification was that a systematic evaluation of the effectiveness of CHX mouthrinses was not available. This may not be correct, since a systematic review, including six-month, randomised clinical trials, was published in 2006, including studies with $0.12 \%$ CHX mouth rinses. ${ }^{3}$

Although the authors aimed to evaluate CHX alone or adjunctive to mechanical control, only adjunctive use was finally assessed. Studies have to recruit gingivitis patients (which the authors identified as a limitation), for a minimum duration of four weeks. This duration was discussed in the paper, since published guidelines recommend a follow up of, at least, six months in order to provide evidence for an antiplaque or antigingivitis effect. ${ }^{4}$

The review was elegantly conducted and reported, following PRISMA guidelines, and the search was performed in three different databases. However, no information was provided on the agreement scores during the process of searching and selection of studies, and only papers in English were included.

The authors selected 34 papers and 13 for meta-analyses. The pooled studies used different concentrations of CHX (ranging from $0.06 \%-0.2 \%$ ), different treatment protocols and different study durations (four weeks to six months). This approach may have led to the significant heterogeneity observed in many meta-analyses.

The meta-analyses were not based in differences between groups in changes baseline-final visit. Instead, the authors calculated differences between groups at baseline and at the final visit. Based on the lack of significant differences at baseline, the authors inferred that the presence of significant differences at the final visit indicated significant differences in the effects. In other systematic review, ${ }^{3}$ with meta-analyses of six RCT of six-month duration evaluating $0.12 \%$ CHX mouth rinses, the changes between baseline-final visit were used, and the reported results [weighted mean difference for plaque $1.040(\mathrm{p}<0.001)$ and for gingival index 0.563 $(p<0.001)$ ] demonstrated a larger effect that the results reported in the commented review.

The conclusion is solidly based on the vast amount of data presented by the authors, both in the papers and in the appendix. However, as described before, the meta-analyses presented some 


\section{PERIODONTOLOGY}

limitations (mainly derived from the lack of information in the selected papers) and some subgroup analyses would have been desirable, especially for different concentrations and follow-ups. This subgroup approach was performed with studies qualified as having low risk of bias. A similar conclusion with regards to staining is missing.

\section{David Herrera}

Section of Periodontics, Faculty of Odontology, University Complutense, Madrid, Spain

1. van der Weijden $F$, Slot DE. Oral hygiene in the prevention of periodontal diseases: the evidence. Periodontol 2000. 2011; 55: 104-123.

2. Schiott CR, Löe H, Jensen SB, Kilian M, Davies RM, Glavind K. The effect of chlorhexidine mouthrinses on the human oral flora. J Periodontal Res 1970; 5: 84-89.

\section{Practice point}

- There is strong evidence for the antiplaque and antigingivitis effects of $\mathrm{CHX}$ mouth rinses used as adjuncts to regular oral hygiene in gingivitis patients

- The use of $\mathrm{CHX}$ is associated with a $33 \%$ plaque and $26 \%$ gingivitis reduction, as compared to controls.

3. Gunsolley JC. A meta-analysis of six-month studies of antiplaque and antigingivitis agents. J Am Dent Assoc 2006; 137: 1649-1657.

4. Guidelines for acceptance of chemotherapeutic products for the control of supragingival dental plaque and gingivitis. Council on Dental Therapeutics. Am Dent Assoc 1986; 112: 529-532.

Evidence-Based Dentistry (2013) 14, 17-18. doi:10.1038/sj.ebd.6400915 